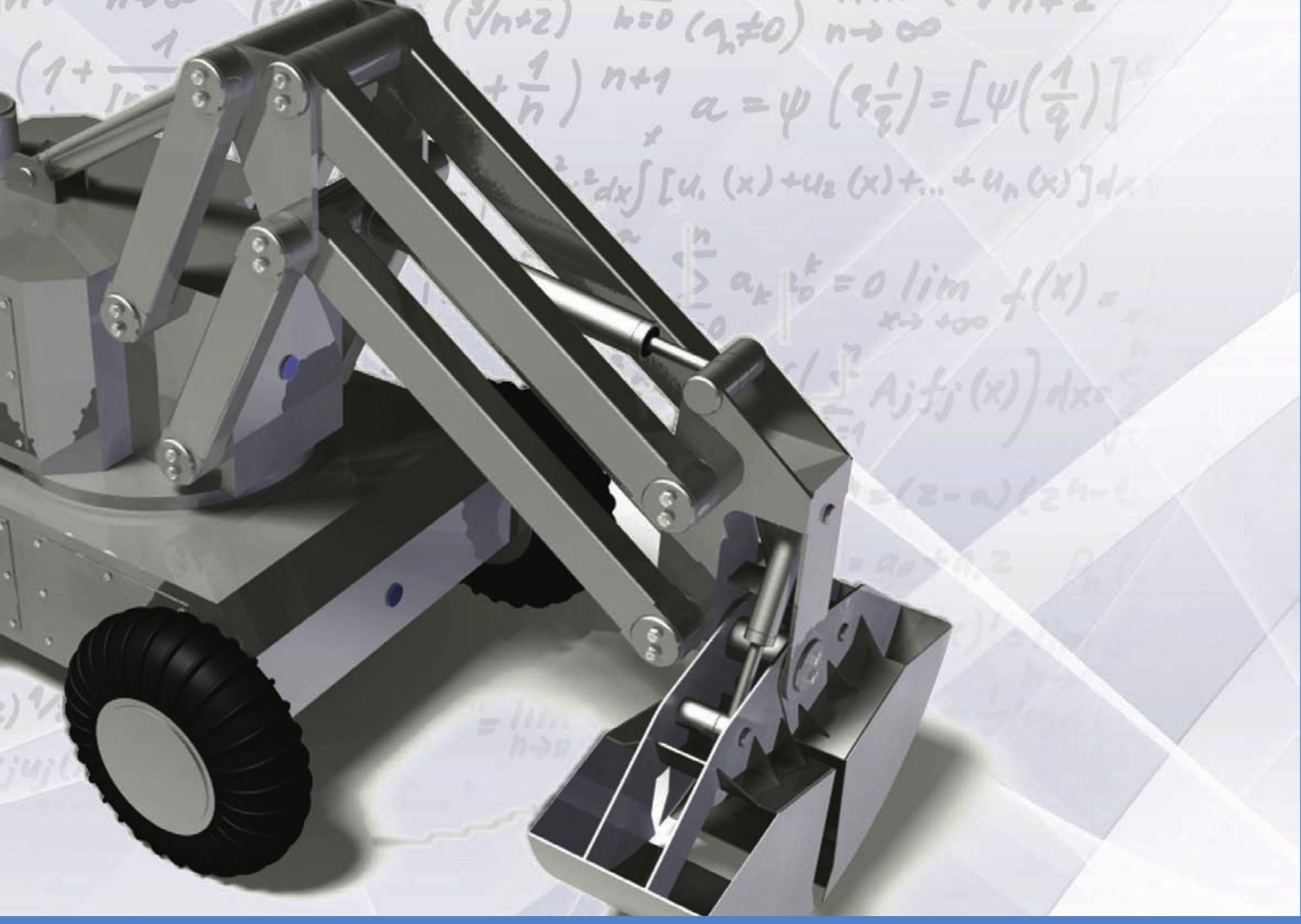

\title{
Modelo \\ matemático de un \\ robot móvil
}

Fausto Freire Carrera

Resumen- Este artículo trata sobre la elaboración de un modelo matemático para un robot móvil de tres ruedas mediante la utilización de las ecuaciones de Appel, en este modelo se tomó en cuenta la cinemática y la dinámica del robot móvil.

En este estudio el robot es analizado como un sistema no-holónomo, lo que permite unificar aspectos cinemáticos, dinámicos, electro-mecánicos y geométricos del robot, en un solo sistema de ecuaciones diferenciales. La mayoría de los modelos utilizan el formalismo estandarizado de Lagrañian para modelar el comportamiento de robots móviles, a pesar que no es aplicable a sistemas con enlaces no diferenciables, siendo una opción la utilización de las ecuaciones de Appel para sistemas no-holónomos.

Palabras clave-- Ecuación de Appel, modelo matemático, robot móvil, sistema mecánico, trayectoria. 
La mayoría de los métodos utilizados para modelar la dinámica de los robots móviles utilizan la metodología mediante la utilización de las ecuaciones de Lagrañian de segundo género, a pesar que existen métodos no menos interesantes como por ejemplo mediante la utilización de las ecuaciones de Appel para sistemas no-holónomos.

La dinámica de los sistemas no-holónomos se utilizan cuando el formalismo estandarizado de Lagrañian no es aplicable a los sistemas con enlaces no diferenciables, es decir las ecuaciones de los enlaces definidas a partir de las coordenadas generalizadas $q_{j}$ y las velocidades generalizadas $q_{j}$ (Borizov, 2002) como:

\section{$f_{i}(q, \dot{q}, t)=0, i=1, \ldots, k, q=\left(q_{1}, \ldots, q_{n}\right)$}

No pueden ser presentadas en forma integral (Herz, 1910) $\quad F_{i}(q, t)=0$, en este caso las ecuaciones de Appel son una solución, las cuales se escriben como:

$$
\left(\frac{\partial S}{\partial \ddot{\mathbf{n}}}\right)^{T}=\mathbf{\Pi},
$$

Donde la función S, es la energía de la aceleración del sistema (Lurye, 1961), calculada tomando en cuenta las ecuaciones de los enlaces, o las funciones de Appel, y $\Pi$ es el vector de las fuerzas generalizadas.

Para modelar el movimiento del robot móvil de ruedas propuesto en este artículo se utilizó un modelo (Martinenko, 2003) donde la posición del robot es determinada mediante siete coordenadas generalizadas que forman el vector $q$ :

$$
\mathbf{q}=\left|x, y, \psi, \beta, \varphi_{1}, \varphi_{2}, \varphi_{3}\right|^{T}
$$

donde $x, y$ son las coordenadas del punto $A$ (que es el centro de la línea que une las ruedas motrices); $\psi$ es el ángulo de giro de la plataforma, contabilizado desde el eje $x ; \beta$ es el ángulo de giro del soporte de la rueda royal en relación a la plataforma, $\Phi 1, \Phi 2, \Phi 3$ ángulos de giro de las ruedas en relación con los ejes horizontales.

El movimiento del robot se analiza en relación a un sistema de coordenadas estacionario OXY. El sistema de las coordenadas móvil $A x_{1} y_{1}$ con centro en el punto $A$, está fijo en plataforma del robot.

El eje $x_{1}$ es perpendicular al trozo que une los centros de las ruedas motrices, y es habitualmente el eje de simetría del robot. La tracción a las ruedas motrices es independiente determinada por los motores de iguales características instalados en las ruedas y son controlados mediante tensiones aplicadas a los motores correspondientemente: $U_{L}$ - para la rueda izquierda y $U_{R}$ - para la rueda derecha. fricción lo que conlleva a cinco ecuaciones independientes de los enlaces no-holónomos. Así, el robot tiene dos grados de libertad y en calidad de las seudo-velocidades $V, \Omega$ es conveniente escoger la relación $\quad V=\dot{x} \cos \psi+\dot{y} \sin \psi$ que es la velocidad del punto A y la velocidad angular de la plataforma $\Omega=\psi$.

El vector de la velocidad del centro de la tercera rueda está en el plano de esta rueda. Esta condición representa el enlace no-holónomo, que se puede escribir mediante la ecuación:

$$
\dot{\beta}+\Omega=\frac{V}{d} \sin \beta-\frac{\Omega b}{d} \cos \beta \text { (2) }
$$

Esta ecuación permite calcular la velocidad angular del soporte de la rueda delantera.

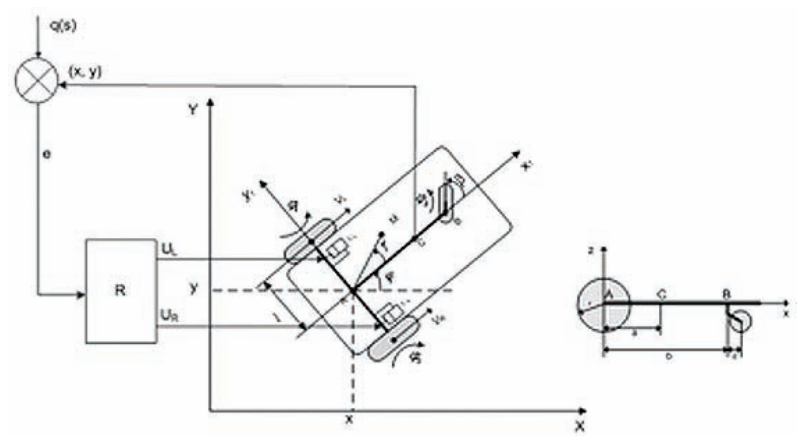

Fígura 1. Esquema del robot móvil

Generalmente la masa de la rueda royal con su soporte son insignificantes en comparación con la masa de la plataforma lo cual al momento de definir la ecuación de Appel no se toma en cuenta.

Con estas consideraciones la expresión de la función de Appel tiene la forma siguiente:

$$
S=\frac{1}{2} m\left[\left(\dot{V}+\Omega^{2} a\right)^{2}+(-\dot{\Omega} a+V \Omega)^{2}\right]+\frac{1}{2} I_{c}\left(\dot{\Omega}^{2}+\Omega^{4}\right)
$$

donde $m$ es la masa de la plataforma del robot, $V$ es la velocidad, $\mathrm{I}^{2}=m \rho^{2}$ es el momento de inercia y $\rho$ es el radio de inercia de la plataforma del robot relativo al eje que pasa por el centro de masas.

La masa de las ruedas motrices incrementa los coeficientes inerciales en la ecuación (3) pero no influyen en la estructura de la función de Appel.

La potencia de los momentos de los motores que actúan sobre las ruedas motrices del robot son:

$$
N=\left(M_{L}, \vec{\omega}_{L}\right)+\left(M_{R}, \vec{\omega}_{R}\right)=\frac{1}{r}\left(M_{L}+M_{R}\right) V+\frac{l}{r}\left(-M_{L}+M_{R}\right) \Omega
$$

donde $\vec{\omega}_{L}, \vec{\omega}_{R}$ son las velocidades angulares de las ruedas motrices, I es la mitad de la distancia entre las ruedas motrices y $r$ es el radio de la rueda motriz. 
Los coeficientes de las seudo-velocidades en la fórmula (4) determinan las fuerzas generalizadas en las ecuaciones de Appel.

$$
\begin{aligned}
& \frac{\partial S}{\partial \dot{V}}=\frac{1}{r}\left(M_{L}+M_{R}\right) \\
& \frac{\partial S}{\partial \dot{S}}=\frac{l}{r}\left(-M_{L}+M_{R}\right)
\end{aligned}
$$

De esta forma en calidad de modelo matemático completo del robot móvil se puede considerar el sistema de ecuaciones diferenciales no lineales siguiente:

$$
\left\{\begin{array}{l}
\dot{x}=V \cos \psi \\
\dot{y}=V \sin \psi \\
\dot{\psi}=\Omega \\
\dot{\varphi}_{3}=\frac{V_{03}}{R_{3}} \\
\dot{\beta}=-\Omega+\frac{V}{d} \sin \beta-\frac{\Omega b}{d} \cos \beta \\
\dot{V}=-\frac{2 c_{2} n^{2}}{m r^{2}} V+a \Omega \Omega^{2}+\frac{c_{1} n}{m r}\left(U_{i}+U_{R}\right) \\
\dot{\Omega}=-\frac{2 c_{2} l^{2} n^{2}}{I_{A} r^{2}} \Omega-\frac{m a}{I_{A}} V \Omega+\frac{c_{1} l n}{I_{A} r}\left(U_{R}-U_{L}\right)
\end{array}\right.
$$

donde $\mathrm{I}_{A}=\mathrm{I}_{C}+m a^{2}$ es el momento de inercia del robot móvil en relación al eje que pasa por el punto $A, V_{03}$ velocidad del centro de la tercera rueda.

Para modelar el comportamiento de los motores de corriente continua nos limitamos al modelo (Devianin 1999) siguiente:

$$
\begin{aligned}
& M_{R}=c_{1} n U_{R}-\frac{c_{2} n^{2}}{r}(V+\Omega l) \\
& M_{L}=c_{1} n U_{L}-\frac{c_{2} n^{2}}{r}(V-\Omega l)
\end{aligned}
$$

donde $C_{1}, C_{2}$ son las características técnicas del electromotor, $U_{L}, U_{R}$ son los voltajes actuantes en el motor izquierdo y derecho, $n$ es la razón de transmisión del reductor.

\section{Simulación de Movimientos Programados}

Movimiento programado es aquel movimiento mediante el cual uno de los puntos del robot (que puede ser un radar u otro dispositivo de control) se mueve por una curva con una velocidad dada. La determinación de los voltajes necesarios para que el robot siga por una determinada trayectoria con cierto grado de exactitud se tratan en muchos trabajos (Martinenko 1999-2002, Kolmanovsky 1995, Samson 1993) y es similar al primer problema de la Dinámica que en la Mecánica de Newton equivale a la definición de las fuerzas por un movimiento conocido de un punto material y que en la Mecánica Analítica de Lagrañían equivale a la construcción de los campos de fuerza por una variedad dada de curvas integrales en el espacio de configuración. Desde el punto de vista matemático estos problemas clásicos se reducen a la obtención de la parte derecha de las ecuaciones diferenciales ordinarias, para las cuales las trayectorias dadas son sus curvas integrales.

Concretamente para el robot de la Figura 1, determinamos un movimiento programado del punto $M$ ubicado en el robot en un sistema de coordenadas fijo, mediante funciones suaves del siguiente tipo.

$$
X_{M}=X_{M}(t), Y_{M}=Y_{M}(t) .
$$

En la Figura 2 tenemos las trayectorias programadas del punto $M$, que se mueve con una velocidad de $V_{M}=1 \mathrm{M} / \mathrm{s}$
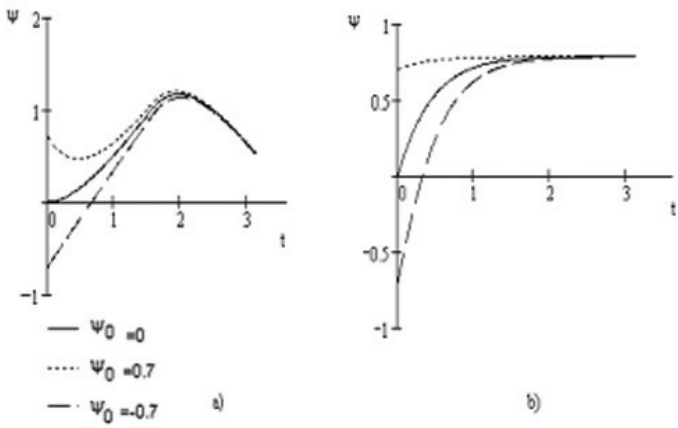

Fígura 2. Trayectoria programadas del punto M:

a- trayectoria compuesta de 2 de circunferencia de $R=1 m$, b- trayectoria lineal.

La proyección de la velocidad del punto M en eje de coordenadas fijo serán funciones continuas del tiempo

$$
\begin{aligned}
& V_{M Y}=V_{M Y}(t)=\dot{Y}_{M} \\
& V_{M X}=V_{M X}(t)=\dot{X}_{M}
\end{aligned}
$$

Algunas características de los problemas que representan sistemas no-holónomos, son las restricciones que presentan al momento de definir trayectorias programadas. En este caso la ecuación de relación $--V_{A X} \sin \psi+V_{A Y} \cos \psi=$ 0 da como resultado la imposibilidad de definir al azar las coordenadas angulares del robot, el ángulo de curso del robot debe ser la resolución de la ecuación diferencial de primer orden:

$$
\frac{d \psi}{d t}=\frac{-V_{M X} \sin \psi+V_{M Y} \cos \psi}{b_{M} \cos \gamma}
$$

donde $b_{M} \cos \gamma$ y $b_{M} \sin \gamma$ son los coordenadas del punto $M$ en el sistema de coordenadas móvil $A x_{1} y_{1}$.
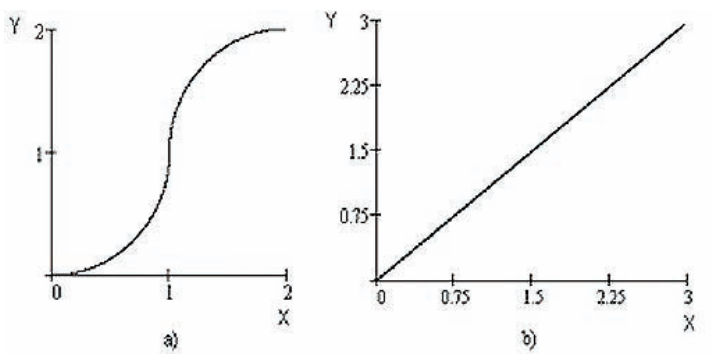

Fígura 3. Relación del ángulo de curso en función del tiempo: a-trayectoria curvilinea, b-trayectoria lineal. 
La integración de la ecuación (10) con condiciones iniciales $\psi(0)=\psi 0$ permite encontrar la ley de cambio del ángulo de curso $\psi$. Y el módulo de la velocidad del punto A se determina mediante la fórmula:

$$
V=\frac{V_{M X} \cos (\psi+\gamma)+V_{M Y} \sin (\psi+\gamma)}{\cos \gamma}
$$

El módulo $V$ de la velocidad del punto $A$ y la velocidad angular del robot deben satisfacer las ecuaciones dinámicas, que se obtienen de reemplazar (7) en (6)

$$
\begin{aligned}
& \dot{V}=-\frac{2 c_{2} n^{2}}{m r^{2}} V+a \Omega^{2}+\frac{c_{1} n}{m r}\left(U_{l}+U_{R}\right) \\
& \dot{\Omega}=-\frac{2 c_{2} l^{2} n^{2}}{I_{A} r^{2}} \Omega-\frac{m a}{I_{A}} V \Omega+\frac{c_{1} l n}{I_{A} r}\left(U_{R}-U_{L}\right)
\end{aligned}
$$

Después de reemplazar (10) y (11) en (12) se puede obtener un sistema de ecuaciones algebraicas (13) de donde encontramos los voltajes $U_{L}$ y $U_{R}$ que corresponden a la realización del movimiento programado (8).

$U_{L}=\frac{-l a \Omega^{2} m r^{2} c_{2}+2 l c_{2}^{2} n^{2} V-c_{1} m a \Omega V r^{2}-2 c_{1} c_{2} n^{2} l^{2} \Omega-c_{1} \dot{\Omega} I_{d} r^{2}+l \dot{V} m r^{2} c_{2}}{l\left(c_{1}+c_{2}\right) c_{1} n r}$

$U_{R}=\frac{-l a \Omega^{2} m r^{2}+2 l c_{2} n^{2} V+l \dot{V} m r^{2}+m a \Omega V r^{2}+2 c_{2} n^{2} l^{2} \Omega+\dot{\Omega} I_{A} r^{2}}{\operatorname{lrn}\left(c_{1}+c_{2}\right)}$

\section{Resultados de la Modelación del Problema Directo}

A continuación se presentarán los resultados de la simulación cuando en punto $M$ se mueve por diferentes trayectorias con una velocidad constante $V_{M}=1 \mathrm{M} / \mathrm{C}$, con una masa de la plataforma del robot $m=12 \mathrm{~kg}$ y con parámetros $r$ $=0,08 \mathrm{~m} ; l=0,2 \mathrm{~m} ; h=0,56 \mathrm{~m} ; \mathrm{cl}, \mathrm{c2}=0,084 \mathrm{H}$.

En las Figuras 4 y 5 se presenta los resultados de la resolución del problema directo para diferentes trayectorias del punto $M$.
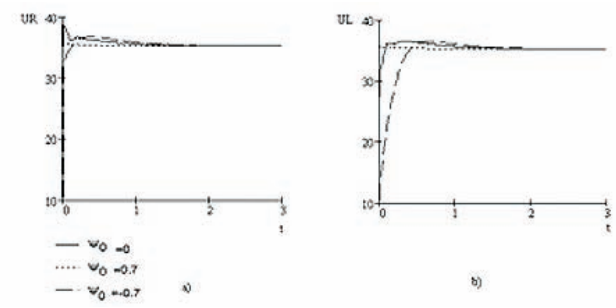

Figura 4. Voltaje en los motores para la trayectoria curvilínea: a-motor derecho; b- motor izquierdo.
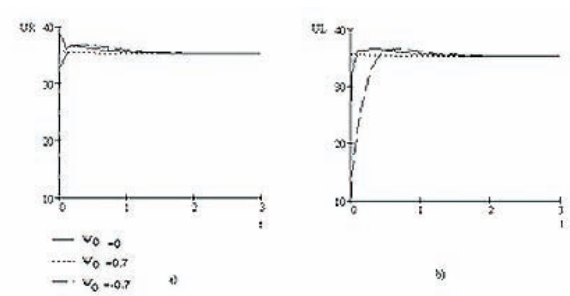

Fígura 5. Voltaje en los motores para la trayectoria rectilínea: a-motor derecho; b- motor izquierdo.

En la Figura 6. se representa la trayectoria del punto $A$ con diferentes valores de ángulo $\Psi_{0}$ en comparación con las trayectorias del punto $M$.
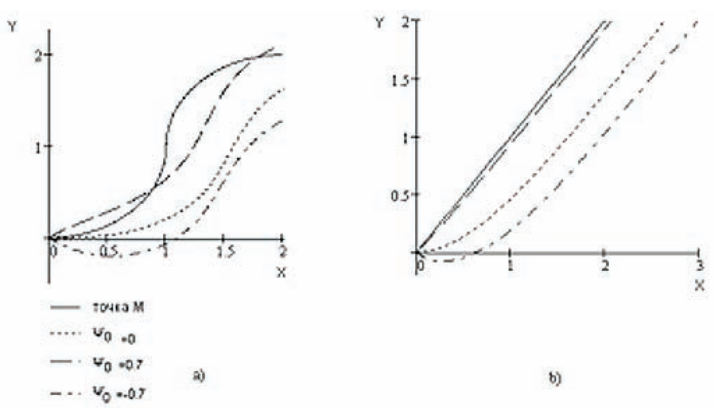

Fígura 6. Trayectoria del punto A con diferentes valores del ángulo: a- trayectoria curvilínea; $b$ - tryectoria líneal.

\section{Conclusiones:}

El modelo matemático obtenido mediante la utilización de las ecuaciones de Appel, permiten de forma clara modelar el movimiento del móvil por varios tipos de trayectorias.

La masa de las ruedas incrementan los coeficientes inerciales pero no afecta en la estructura de las ecuaciones de Appel.

Algún grado de dificultad impone determinar la función de la energía de la aceleración del sistema.

Dependiendo de las trayectorias que a de seguir el robot se definen las secuencias de voltajes enviados a los motores del robot.

Para la resolución analítica de las ecuaciones del modelo matemático del móvil se utilizó el paquete computacional MathCad, lo que permitió ahorrar tiempo, y tener una idea clara acerca del posible del móvil al seguir las trayectorias rectilínea y curvilínea. 


\section{Bibliografía}

- Борисов А. В., Мамаев И. С. (2002). Неголономные динамические системы. Интегрируемость, хаос, странные аттракторы / Сборник статей. - Москва-Ижевск: Институт компьютерных исследований, , 328 стр.

- Девянин Е.А. (1998). О движении колесных роботов. В сб.: Мобильные роботы и мехатронные системы. Докл. науч. школы-конф., М., Изд-во НИИ механики МГУ, 1999, п̃. 169-200.

• Hertz H. (1910). Hertz H. Die Prinzipient der Mechanik in neuem Zusammenhange clargestellt. Ges. Werke, Bd. 3, Leipzig, Barth., 3129.

- Kolmanovsky V.B.\&McClamroch N.H. (1995). Developments in non-holonomic control problem. IEEE Control Systems, v. 15, № 6, p. 20-36

- Лурье А.И. (1961).Аналитическая механика. М., Физматгиз, 824 c.

- Мартыненко Ю.Г. (2000). Динамика мобильных роботов // Соросовский образовательный журнал, т. 6, №5, с. 110-116. Мартыненко Ю.Г. 2002. Проблемы управления и динамики мобильных роботов. Новости искусственного интеллекта, № 4 (52), с. 18-23.

- Мартыненко Ю.Г.\&Кобрин А.М. (1999). Управление автономным движением мобильного робота МЭИ. В сб.

- Мобильные роботы и мехатронные системы. Докл. науч. школы-конф., 7-8 дек. М., Изд-во Ин-та механики МГУ, с. 58-80.

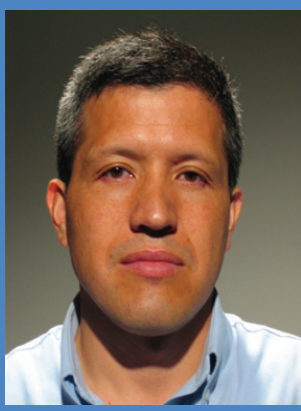

Autor: Fausto Rodrigo Freire Carrera

ffreire@ute.edu.ec

Título: Ingeniero en Sistemas.

Institución: Universidad Técnica Estatal de Kursk - UTEK (Federación de Rusia).

Facultad: Ingeniería en Sistemas, Computación y Automatización.

Título: Máster en Ciencias.

Institución: Universidad Técnica Estatal de Kursk (Federación de Rusia).

Facultad: Facultad de Ingeniería en Sistemas, Computación y Automatización.

Título: Máster en Tecnologías de la Información para la Fabricación.

Institución: Universidad Politécnica de Madrid (España).

Nombre del programa académico: Departamento de Sistemas Inteligentes Aplicados.

Título: Especialista en Robótica.

Institución: Universidad Politécnica de Madrid (España).

Nombre del programa académico: Departamento de Sistemas Inteligentes Aplicados.

Título: Ph.D en Dinámica, Resistencia, Aparatos y Dispositivos Institución: Universidad Técnica Estatal de Kursk (Federación de Rusia).

Nombre del programa académico: Facultad de Mecánica Teórica y Mecatrónica. 